\title{
A Low-Complexity ML Channel Estimator for OFDM
}

\author{
Luc Deneire, Member, IEEE, Patrick Vandenameele, Member, IEEE, Liesbet van der Perre, Bert Gyselinckx, and \\ Marc Engels, Member, IEEE
}

\begin{abstract}
Orthogonal frequency-division multiplexing with cyclic prefix enables low-cost frequency-domain mitigation of multipath distortion. However, to determine the equalizer coefficients, knowledge of the channel frequency response is required. While a straightforward approach is to measure the response to a known pilot symbol sequence, existing literature reports a significant performance gain when exploiting the frequency correlation properties of the channel. Expressing this correlation by the finite delay spread, we build a deterministic model parametrized by the channel impulse response and, based on this model, derive the maximum-likelihood channel estimator. In addition to being optimal (up to the modeling error), this estimator receives an elegant time-frequency interpretation. As a result, it has a significantly lower complexity than previously published methods.
\end{abstract}

Index Terms-Equalizers, orthogonal frequency-division multiplexing (OFDM).

\section{INTRODUCTION}

$\mathbf{O}$ RTHOGONAL frequency-division multiplexing (OFDM) has become increasingly popular during the last decades, mainly because it provides a substantial reduction in equalization complexity compared to classical modulation techniques. Indeed, OFDM with cyclic prefix (CP) can be equalized by a single low-rate complex multiplication on each carrier. For this reason, it has been adopted in the upcoming standards for high data-rate wireless networks, such as ETSI Hiperlan II and IEEE 802.11a. These standards use some "zero-carriers" for spectral shaping; for example, some carriers are not used to allow smooth decaying of the spectral power on the border of the bandwith. Hence, these standards (and possible variations) will be referred to as spectral shaping systems.

As opposed to former standards using OFDM modulation, the new standards rely on coherent quadrature amplitude modulation (QAM), and thus require channel estimation. Hence, the complexity of channel estimation is of crucial importance, especially for time-varying channels, where it has to be performed periodically or even continuously.

Existing literature recognizes that, due to the structure of OFDM signals, the channel can be estimated by using the

Paper approved by R. S. Cheng, the Editor for CDMA and Multiuser Communications Systems of the IEEE Communications Society. Manuscript received July 7, 2000; revised April 15, 2002. This paper was presented in part at the IEEE International Conference on Communications, Helsinki, Finland, 2001

L. Deneire is with the University of Nice Sophia-Antipolis, F-06903 SophiaAntipolis, France (e-mail: deneire@i3s.unice.fr).

P. Vandenameele is with Resonext Communications, 3001 Leuven, Belgium (e-mail: patrick.vandenameele@ resonext.com).

L. van der Perre, B. Gyselinckx, and M. Engels are with IMEC, 3001 Leuven, Belgium (e-mail: vdperre@imec.be; gyselinc@imec.be; engelsm@imec.be).

Digital Object Identifier 10.1109/TCOMM.2003.809234 time and frequency correlation of the channel. This frequency correlation has inspired three different approaches. Edfors et al. [1] use the frequency correlation explicitly and derive a linear minimum mean-squared error (LMMSE) estimator. Using optimal rank reduction, they develop a low-complexity algorithm which computes an approximated LMMSE estimator. This approximation is limiting the performance at high signal-to-noise ratios (SNRs). Raleigh and Jones [2] link the frequency correlation to the maximum delay spread and estimate the channel from a part of the carriers only. These carriers must be regularly spaced, which limits the application of their method. Vandenameele et al. [3] also use the length of the channel impulse response explicitly and derive an $a d$ hoc constrained least-squares estimator. Their method allows nonregular spacing of the carriers, with a small limitation on their number (there should be at least as many nonpilot carriers as the length of the channel impulse response).

To avoid the shortcomings of aforementioned methods (high SNR performance limitation for LMMSE, limitation on the spacing/number of pilots for the others), we use the finite delay spread of the channel and develop a low-complexity algorithm capable of estimating the channel from part of the carriers only. We introduce a deterministic model and derive the associated maximum-likelihood (ML) estimator. This ML estimator can be interpreted as a transformation from frequency domain to time domain and back to frequency. The actual estimation is done in the time domain, where the number of parameters (i.e., the channel length) is small. The estimator is obtained by minimizing a quadratic criterion, which, combined with the small number of parameters, leads to a low-complexity algorithm. As such, we have obtained an exact low-complexity solution. We extend our approach to pilot symbol-assisted modulation (PSAM) and link it to the constrained least squares (CLS) solution proposed in [3]. It is worth noting that although our estimator is based on a parametric model, the only parameter is the channel length (by channel, we mean the concatenation of the front-end filters and the propagation channel). The only condition is then that the global channel length (which can be significantly longer than the propagation channel itself) has to be smaller than this one parameter. As a result, the method is rather robust against channel modeling errors, the main disadvantage being that the length parameter of the ML estimator can be rather large, leading to a loss in performance.

After introducing the OFDM system model in Section II, we present the statistical model and channel estimator in Section III, along with the extension to PSAM and the link with the CLS estimator. The time-frequency interpretation of the ML estimator is given, and the benefits provided by a combined PSAM/decision-feedback (DF) system are indicated. 


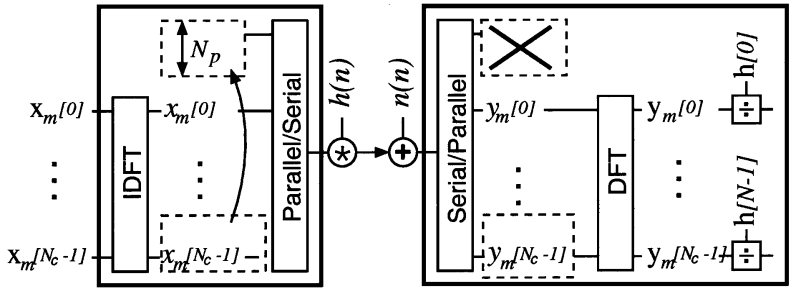

Fig. 1. OFDM system.

Section IV analyzes the complexity of the algorithm, while Section V presents and discusses simulation results for indoor PSAM and spectral-shaping systems.

\section{SYSTEM MODEL}

\section{A. Notations}

Normal letters represent scalar quantities, boldface represents vectors, and boldface capitals represent matrices. Slanted (resp. roman) letters indicate time (resp. frequency) domain quantities. $\mathbf{X}^{T}, \mathbf{X}^{H}$, and $\mathbf{X}^{\#}$, respectively, mean transpose, conjugate transpose, and Moore-Penrose pseudoinverse of $\mathbf{X}$. If $\mathbf{X}$ is full column rank, then $\mathbf{X}^{\#}=\left(\mathbf{X}^{H} \mathbf{X}\right)^{-1} \mathbf{X}^{H}$ and $\mathbf{P}_{X}=$ $\mathbf{X}\left(\mathbf{X}^{H} \mathbf{X}\right)^{-1} \mathbf{X}^{H}$ is the orthogonal projection onto the space spanned by the columns of $\mathbf{X}$.

\section{B. Transmission Model and Training Setup}

OFDM modulation consists in multiplexing QAM data symbols over a large number of orthogonal carriers. To this end, the QAM symbols of an OFDM symbol are passed through an inverse fast Fourier transform (IFFT). In the presence of a time-dispersive channel, a $\mathrm{CP}$ is prepended to each OFDM symbol to preserve orthogonality between carriers and eliminate intersymbol interference (ISI) (for a global overview, see [4]).

We consider a single user/single channel communication setup (see Fig. 1), with OFDM modulation, described by $\mathbf{Y}=\mathbf{X} \odot \mathbf{H}+\mathbf{N}$, where $\odot$ denotes Hadamard (i.e., element-wise) product of the columns of $\mathbf{X}$ with $\mathbf{H}$. For a single OFDM symbol, it boils down to

$$
\mathbf{y}=\mathbf{x} \odot \mathbf{H}+\mathbf{n} .
$$

Notations in the model are detailed here: The QAM source is written as $\mathbf{X}=\left[\mathbf{x}_{0} \cdots \mathbf{x}_{M-1}\right]$, where $\mathbf{x}_{m}=$ $\left[x_{0, m} \cdots x_{N_{c}-1, m}\right]^{T}$ is an OFDM symbol. $N_{c}$ denotes the number of carriers, $m$ is a time index (often omitted for clarity), and $M$ is the number of OFDM symbols. After IFFT and CP insertion, the transmitted signal is $\boldsymbol{X}_{m}=\left[\boldsymbol{X}_{N_{c}-N_{p}, m} \cdots \boldsymbol{X}_{N_{c}-1, m} \boldsymbol{X}_{0, m} \cdots \boldsymbol{X}_{N_{c}-1, m}\right]^{T}$, where $N_{p}$ is the size of the prefix and $\boldsymbol{X}=\left[\boldsymbol{X}_{0} \cdots \boldsymbol{X}_{M-1}\right]$.

For a channel $h=\left[h_{0} \cdots h_{N_{h}-1}\right]$, where $N_{h} \leq N_{p}$, the received vector is, after prefix removal and FFT, $\mathbf{y}_{m}=\mathbf{x}_{m} \odot \mathbf{H}$, where $\mathbf{H}=\left[H_{0} \cdots H_{N c-1}\right]^{T}$ is the FFT of the channel. Equation (1) further takes the additive (possibly colored) Gaussian noise into account. Equalization is then done by a complex division on each carrier.

Two types of training (pure PSAM and spectral-shaping systems) are considered. In classical training-based estimation, all components of $\mathbf{x}$ are known. Spectral-based systems use a minor modification of the classical training, zeroing a small number of carriers (named zero carriers) at the edges and in the middle of the band used. PSAM, on the other hand, bases its channel estimation on a small fraction of the carriers, usually evenly spaced on the whole band, and possibly on varying positions from one OFDM symbol to the next, which allows adapting the channel estimate continuously at the cost of a small overhead. Note that PSAM and spectral shaping are usually combined in bandlimited systems.

\section{ML ESTIMATION}

The ML estimator is first derived, based on a reduced-order model. It is then extended to PSAM, linked to the CLS method, and interpreted in terms of time-frequency transformations. Finally, it is also applied on a combination of PSAM and DF estimation.

\section{A. Reduced-Order Model}

As (1) shows, the OFDM system can be described as a set of parallel Gaussian channels. Because the time-domain channel $\boldsymbol{h}$ has a finite length (smaller than the prefix length in a well-designed OFDM system), these parallel channels feature correlated attenuations. Considering, without loss of generality, $\mathbf{x}=$ $\left[\begin{array}{llll}1 & 1 & \cdots & 1\end{array}\right]^{T}$, the model expressed in (1) becomes

$$
\mathbf{y}=\mathbf{F}\left[\begin{array}{c}
\boldsymbol{h} \\
0
\end{array}\right]+\mathbf{n}=\mathbf{H}+\mathbf{n}
$$

where $\mathbf{F}$ is a $N_{c} \times N_{c}$ FFT matrix. The vector $\mathbf{y}$ is a Gaussian random variable with mean $\mathbf{F}[\boldsymbol{h} 0]^{T}$ and covariance $\mathbf{C}_{n n}$. However, the signal part of $\mathbf{y}$ is contained only in the space spanned by its mean. Separating the "signal subspace" from the "noise-only subspace," the received signal can be rewritten as

$$
\mathbf{y}=\left[\mathbf{F}_{h} \mathbf{F}_{n}\right]\left[\begin{array}{c}
\boldsymbol{h} \\
0
\end{array}\right]+\mathbf{n} .
$$

Relying on this, the reduced space signal is defined as

$$
\boldsymbol{r}=\mathbf{F}_{h}^{\#} \mathbf{y}=\boldsymbol{h}+\mathbf{F}_{h}^{\#} \mathbf{n}=\boldsymbol{h}+\boldsymbol{v}
$$

where $\boldsymbol{v}$ is a zero-mean Gaussian noise of covariance $\mathbf{C}_{v v}=$ $\mathbf{F}_{h}^{H} \mathbf{C}_{n n} \mathbf{F}_{h}$. If $\mathbf{C}_{n n}=\sigma_{n}^{2} I_{N_{c}}, \boldsymbol{v}$ is a white Gaussian noise of covariance matrix $\sigma_{n}^{2} I_{N_{h}}$. The reduced space (Gaussian) signal has a log-likelihood function expressed by

$\log f(\boldsymbol{r})=-\log \left(\pi \operatorname{det}\left(\mathbf{C}_{v v}\right)\right)-\left(\mathbf{F}_{h}^{\#} \mathbf{y}-\boldsymbol{h}\right)^{H} \mathbf{C}_{v v}^{-1}\left(\mathbf{F}_{h}^{\#} \mathbf{y}-\boldsymbol{h}\right)$.

Maximizing this $\log$-likelihood with respect to $\boldsymbol{h}$ leads to the ML estimator given by

$$
\widehat{\mathbf{H}}=\mathbf{F}_{h} \mathbf{F}_{h}^{\#} \mathbf{y}=\mathbf{P}_{\mathbf{F}_{h}} \mathbf{y}
$$

where $\mathbf{P}_{\mathbf{F}_{h}}$ denotes the orthogonal projection on the column space of $\mathbf{F}_{h}$. Before performing the ML estimation, $N_{h}$ must be determined, and we denote its estimation as $N_{\hat{h}}$.

\section{B. Extensions to PSAM and Spectral-Shaping Systems}

In the case of PSAM and spectral-shaping systems, not all symbols in $\mathrm{x}$ are known, and only a subset of $N_{u}$ measured 
carriers can be used. Only this part of the signal (noted $\mathbf{y}_{u}$ ) will be used, and the reduced space signal (4) becomes

$$
\boldsymbol{r}=\mathbf{F}_{u h}^{\#} \mathbf{y}_{u}=\boldsymbol{h}+\mathbf{F}_{u h}^{\#} \mathbf{n}=\boldsymbol{h}+\boldsymbol{v}
$$

where $\mathbf{F}$ has been decomposed as

$$
\mathbf{F}=\left[\begin{array}{c}
\mathbf{F}_{u h} \mid \\
\hline \mathbf{F}_{l h} \mid
\end{array}\right]
$$

and where measured pilots have been grouped together. Ungrouped pilots can be handled by straightforward permutations in columns and lines of the vectors and matrices.

The ML estimator for spectral-shaping systems, corresponding to (7), is $\widehat{\mathbf{H}}_{u}=\mathbf{P}_{\mathbf{F}_{u h}} \mathbf{y}_{u}$ (only the measured carriers are estimated, as they are the only ones carrying data) and, for PSAM, it is $\widehat{\mathbf{H}}=\mathbf{F F}_{u h}^{\#} \mathbf{y}_{u}$ (the whole channel is estimated).

\section{Link With CLS}

Vandenameele et al. [3] derived a CLS estimator by expressing the reduced channel length in the following equation:

$$
\left[\frac{\boldsymbol{h}}{0}\right]=\left[\overline{\mathbf{C}_{u} \mid \mathbf{C}_{l}}\right] \cdot\left[\frac{\mathbf{y}_{u}}{\mathbf{y}_{l}}\right]
$$

where $\mathbf{C}_{u}$ and $\mathbf{C}_{l}$ are parts of an IFFT matrix. From this equation, we can write a constraint equation as

$$
\mathbf{C}_{u} \mathbf{y}_{u}+\mathbf{C}_{l} \mathbf{y}_{l}=0
$$

leading to the CLS solution

$$
\widehat{\mathbf{H}}_{u}=\mathbf{C}_{u}^{H}\left(\mathbf{C}_{u} \mathbf{C}_{u}^{H}\right)^{\#} \mathbf{C}_{l} \mathbf{C}_{l}^{H}\left(\mathbf{C}_{l} \mathbf{C}_{l}^{H}\right)^{\#} \mathbf{C}_{u} \mathbf{y}_{u} \triangleq \mathbf{P}_{S} \mathbf{y}_{u}
$$

where $\mathbf{P}_{S}$ is an orthogonal projection matrix (Hermitian and such that $\mathbf{P}_{S}^{2}=\mathbf{P}_{S}$ ) which can be further simplified to $\mathbf{P}_{S}=$ $\mathbf{C}_{u}^{\#} \mathbf{P}_{\mathbf{C}_{l}} \mathbf{C}_{u}$. However, finding an explicit expression for $\mathbf{S}$ is not straightforward.

When $N_{c}-N_{u} \geq N_{\hat{h}}$ (otherwise $\mathbf{P}_{S}$ is not full rank and the CLS solution is not defined) and taking into account that $\mathbf{C}_{u} \mathbf{F}_{u h}+\mathbf{C}_{l} \mathbf{F}_{l h}=0$, the following equations show that $\mathbf{P}_{S}=$ $\mathbf{P}_{\mathbf{F}_{u h}}$. Indeed, the combined projection on $\mathbf{S}$ and $\mathbf{F}_{u h}$ is shown to be a projection on $\mathbf{F}_{u h}$

$$
\begin{aligned}
\mathbf{P}_{S} \cdot \mathbf{P}_{\mathbf{F}_{u h}} & =\mathbf{C}_{u}^{\#} \mathbf{P}_{\mathbf{C}_{l}} \mathbf{C}_{u} \mathbf{F}_{u h}\left(\mathbf{F}_{u h}^{H} \mathbf{F}_{u h}\right)^{-1} \mathbf{F}_{u h}^{H} \\
& =-\mathbf{C}_{u}^{\#} \mathbf{P}_{\mathbf{C}_{l}} \mathbf{C}_{l} \mathbf{F}_{l h} \mathbf{F}_{u h}^{\#} \\
& =\mathbf{C}_{u}^{\#} \mathbf{C}_{l} \mathbf{F}_{l h} \mathbf{F}_{l h}^{\#} \mathbf{C}_{l}^{\#} \mathbf{C}_{u} \\
& =\mathbf{C}_{u}^{\#} \mathbf{C}_{u} \mathbf{F}_{u h} \mathbf{F}_{u h}^{\#} \mathbf{C}_{u}^{\#} \mathbf{C}_{u} \\
& =\mathbf{P}_{\mathbf{F}_{u h}} .
\end{aligned}
$$

Hence, the two spaces are the same and the estimators are identical.

\section{Time-Frequency Interpretation}

As $\widehat{\mathbf{H}}=\mathbf{F}\left(\mathbf{F}_{u h}^{H} \mathbf{F}_{u h}\right)^{-1} \mathbf{F}_{u h}^{H} \mathbf{y}_{u}$, the channel estimator is the cascade of a partial IFFT, a weighting matrix, and a partial FFT. Indeed, if all pilots are present, or if they are regularly spaced, this boils down to going from the frequency domain to the time

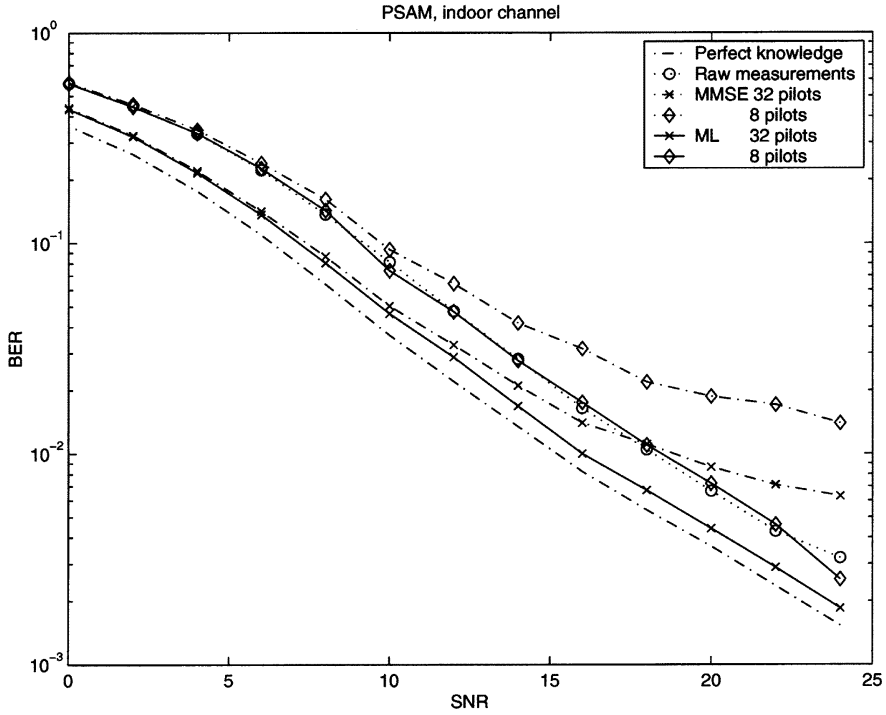

Fig. 2. Comb spectrum for $N_{c} / N_{\hat{h}}=8$.

domain, forcing the time channel estimator to be of length $N_{\hat{h}}$, and going back to the frequency domain [2]. For an arbitrary number of pilots $\left(\geq N_{\hat{h}}\right)$, the same global scheme is applicable, with the following modifications.

- The initial IFFT is partial, as only part of the carriers are measured.

- The nontrivial part of the channel impulse response is weighted by $\left(\mathbf{F}_{u h}^{H} \mathbf{F}_{u h}\right)^{-1}$.

\section{E. Combination of PSAM and DF}

The classical ML solution can be applied to a combination of PSAM and DF. Indeed, suppose we use the pilot symbols along with decisions taken on the other carriers, then $\boldsymbol{r}=\boldsymbol{h}+\boldsymbol{v}$ remains valid, with a given $\mathbf{C}_{v v}$, which leads to $\widehat{\mathbf{H}}=\mathbf{P}_{\mathbf{F}_{h}} \mathbf{y}$. Hence, if the designer can afford the increment in complexity, the combination of PSAM and DF is desirable. Indeed, Fig. 2 shows that a difference in performance of $2-3 \mathrm{~dB}$ can be expected between an all-pilot system (which is equivalent to combined PSAM/DF if decision errors are neglected) and a PSAM system with eight pilot carriers.

\section{COMPLEXITY}

The complexity of the ML estimator is significantly lower than [1], both for spectral shaping and PSAM systems. This low complexity relies on the time-frequency interpretation and pruning of the (I)FFTs.

\section{A. Spectral-Shaping Systems}

By construction, $\mathbf{P}_{\mathbf{F}_{u h}}$ is a low-rank matrix (of rank $N_{\hat{h}}$ ). Taking its Hermiticity into account, it can be written as

$$
\mathbf{P}_{\mathbf{F}_{u h}}=V V^{H}
$$

where $V$ is a matrix of size $N_{c} \times N_{\hat{h}}$ that can be precomputed. Hence, the complexity for computing the estimator is $2 N_{u} \times$ $N_{\hat{h}}$ complex multiplications for the global ML estimator. This complexity is about the same as for [1]; however, while [1] uses an approximation, (13) is exact. 


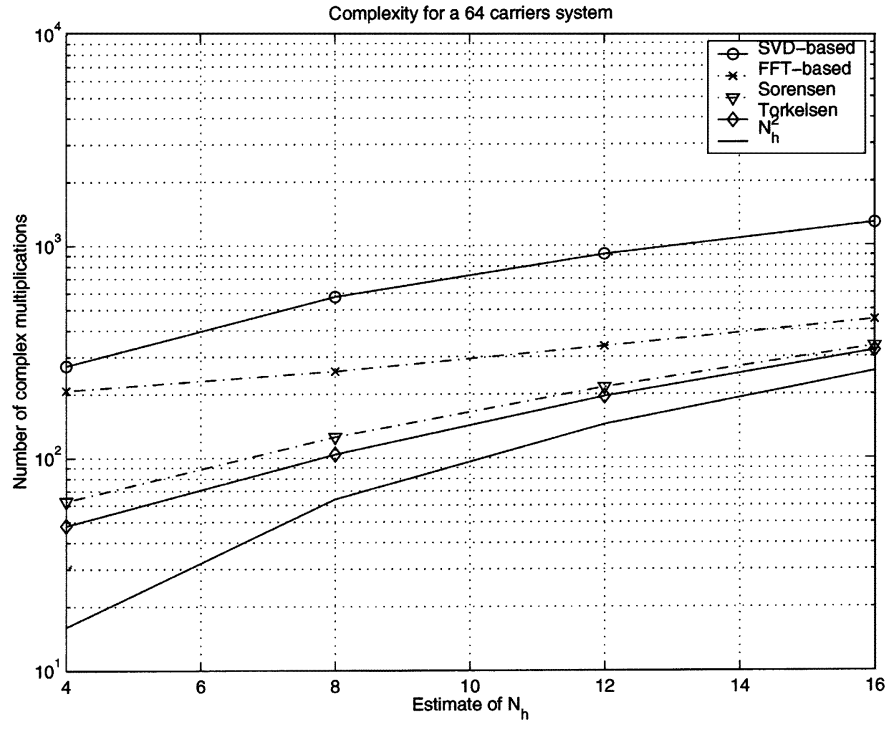

Fig. 3. FFT-based approaches outperform the SVD-based approaches by an order of magnitude for spectral-shaping systems.

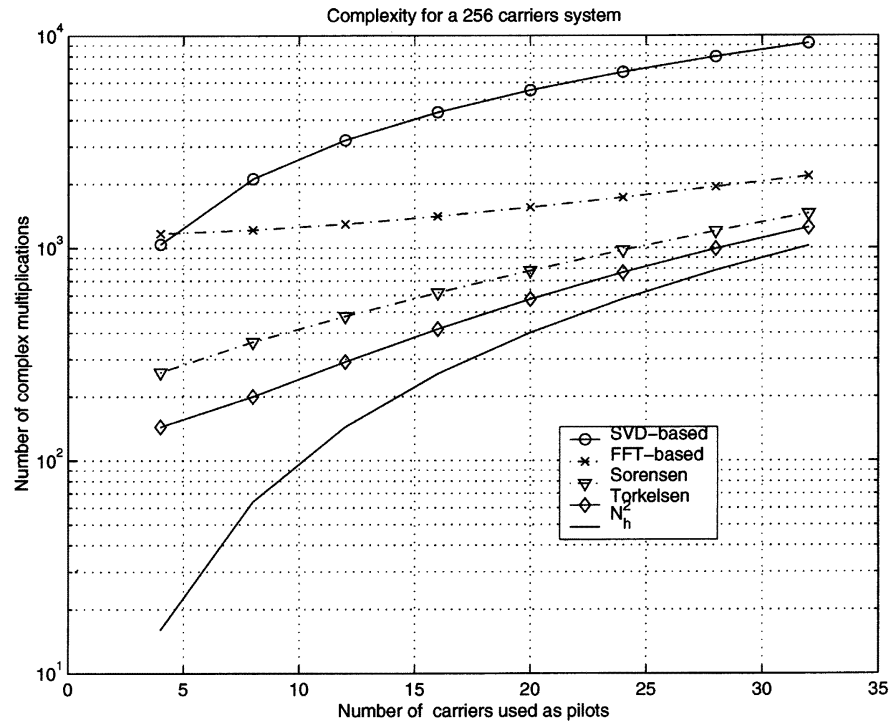

Fig. 4. FFT-based approaches outperform the SVD-based approaches by an order of magnitude for PSAM-based systems.

Further complexity reduction can be obtained by using the time-frequency interpretation. Indeed, the projection operation can be expressed by the cascade of two partial FFTs, weighted by an $N_{\hat{h}} \times N_{\hat{h}}$ matrix (if all carriers are used as pilots, it is an identity matrix). With a radix-4 implementation of the FFT, the complete estimator would require $1.5 N_{c}\left(\log _{4}\left(N_{c}\right)-1\right)+N_{\hat{h}}^{2}$ complex multiplications. Furthermore, some additional complexity gain can be achieved by using FFT pruning or transform decomposition [5]. Such techniques lead to a significant gain for the Fourier transforms. However, the last term $\left(N_{\hat{h}}^{2}\right)$, due to the weighting matrix $\left(\left(\mathbf{F}_{u h}^{H} \mathbf{F}_{u h}\right)^{-1}\right)$, remains unchanged (Figs. 3 and 4).

\section{B. PSAM}

When using PSAM, a comb spectrum (Fig. 5) has to be measured, and only the teeth of this comb are used for the FFTs. This particular case has been studied by $\mathrm{He}$ and Torkelson [6]. In this case, the DFT can be computed with $N_{c} / 4+N_{\hat{h}} / 2+\log _{2} N_{\hat{h}}-$ $N_{\hat{h}}$ complex multiplications, which represents a large gain for a large number of carriers.

Figs. 3 and 4 show complexity evaluations of the four algorithmic approaches:

- singular value decomposition (SVD)-based approach of [1];

- frequency-time approach with plain FFTs;

- frequency-time approach with FFT pruning like Sorensen [5];

- frequency-time approach with FFT optimized for a comb spectrum (He and Torkelson [6]).

The complexity for FFT-based solutions is much lower than for the SVD-based approach, both for spectral shaping and PSAM systems. Futhermore, simulations (Section V) show that the ML algorithm can work with a significantly smaller $N_{\hat{h}}$ than the LMMSE, which results in a still larger gain than appears in Fig. 3.

For a relatively large number of pilot carriers, the main contribution to the complexity is due to the weighting matrix (see the $N_{\hat{h}}^{2}$ curves in Figs. 3 and 4). However, for pure PSAM with regularly spaced pilot carriers, it can easily be shown that the weighting matrix $\left(\mathbf{F}_{u h}^{H} \mathbf{F}_{u h}\right)^{-1}$ is proportional to the identity matrix, and complexity is even lower. This special case of our algorithm is the frequency correlation part of the algorithm developed by Raleigh and Jones [2].

\section{Simulation Results}

To evaluate the performances of the ML estimator, and compare it with the LMMSE algorithm, we simulate a spectralshaping system and a PSAM-based system in an indoor radio channel.

Two OFDM schemes with 64 and 256 carriers are considered, both with uncoded quaternary phase-shift keying (QPSK) modulated carriers and a $\mathrm{CP}$ of 16 . The 64-carriers scheme is simulated with the Hiperlan II zero carriers, and the 256 carriers with PSAM. The data rate is $25 \mathrm{Msamples} / \mathrm{s}$ over the air (i.e., including the $\mathrm{CP}$ ) with a carrier frequency of $5.6 \mathrm{GHz}$.

We consider a collection of 120 indoor office-like channels. The channel is modeled by means of a ray-tracing technique, considering 20 emitter locations and six receiver locations. From the ray-tracing results, it appears that the channel length is of the order of four to six, and can be modeled as having an exponentially decaying power delay profile with normalized time constant two (for simulation of [1]). Note that in these simulations, the channel was not formed exactly by six rays that were sample-spaced, but rather by about two-three rays, nonsample-spaced, and then resampled. The influence of the front-end filters, which significantly lengthen the overall channel, was not taken into account, except in Fig. 6.

For the spectral shaping system, the bit-error rate (BER) is simulated for both LMMSE [1] and ML estimators and for $N_{\hat{h}}$ ranging from 4 to 16 . BER based on exact channel knowledge and raw measurements are evaluated for comparison. Simulation results (Fig. 7) clearly show that the LMMSE suffers from a threshold effect at high SNR, as reported in [1]. To obtain sim- 


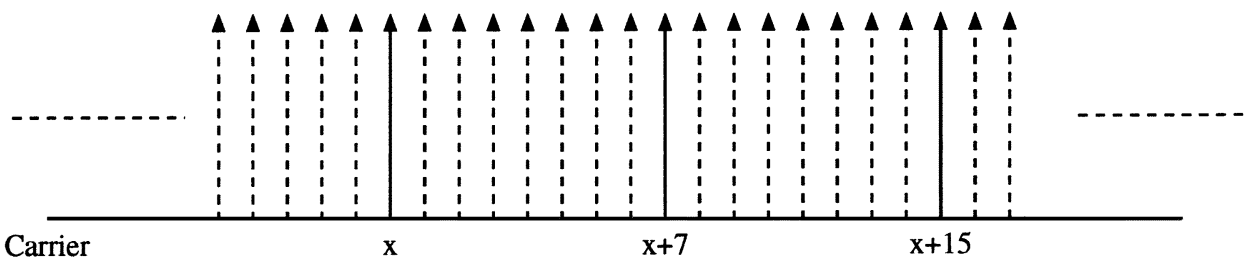

Fig. 5. ML estimator behaves better than LMMSE for low-dimension spaces.

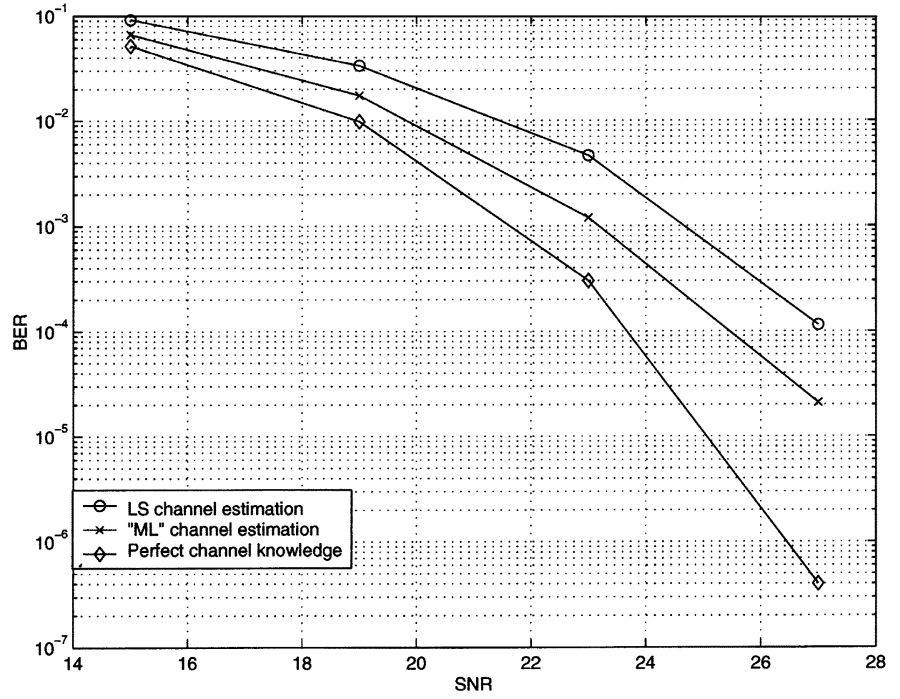

Fig. 6. ML estimator behaves better than LMMSE for PSAM with a small number of pilots.

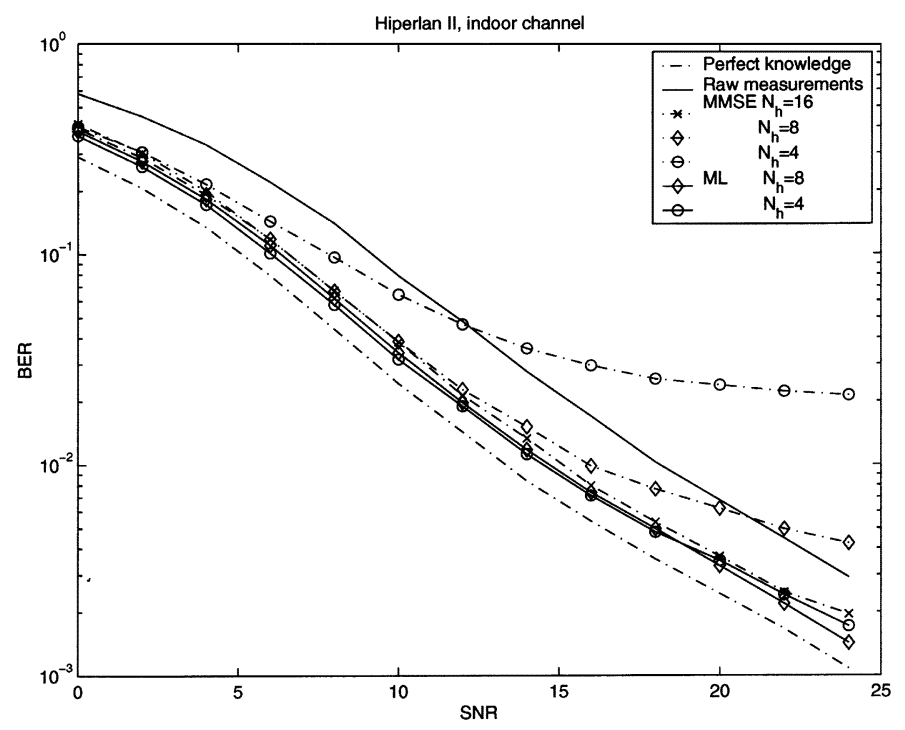

Fig. 7. ML estimator with an expected channel length of 16 still enhances the reception by $2 \mathrm{~dB}$ with real front-end filters and nonsample-spaced channels.

ilar performances for both algorithms, $N_{\hat{h}}$ must be two to four times larger for LMMSE than for ML.

For the PSAM-based system, we have evaluated both estimators with 8 and 32 tones and $N_{\hat{h}}=8$ (Fig. 2). In this simulation, "raw measurements" mean measurements on all pilots. As for the spectral-shaping system, the flooring effect of the LMMSE estimator is essentially limiting its effectiveness at high SNRs. Noteworthy, the ML estimator based on eight over 256 tones gives similar performance as raw measurements on all carriers.
As with all parametric model-based estimators, our estimator can have problems in the presence of modeling errors. This has already been partially highlighted in the previous figures, which were based on a nonsample-spaced channel, and with different expected lengths of channel. To further assess our methodology, we have simulated a "real" front-end filter, as designed in [7], consisting of a Butterworth filter, which is an equivalent baseband tenth-order low-pass infinite impulse response (IIR) filter with $-3 \mathrm{~dB}$ at $10 \mathrm{MHz}$, maximal passband ripple $=0.05 \mathrm{~dB}$ (between $0-8.3 \mathrm{MHz}$ ), and a stopband suppression of $40 \mathrm{~dB}$ ( $>20 \mathrm{MHz}$ ). Obviously, for such a long filter, the equivalent channel length is much larger than four to six taps, and we have chosen an expected length equal to the CP length (16). The simulations have been conducted for a QAM-64 constellation and a sampling rate of $80 \mathrm{MHz}$, with perfect channel knowledge, with our ML channel estimator and with the classical least-squares (LS) estimator. Simulations show that, even with such a long front-end filter, there is still about $2 \mathrm{~dB}$ to be gained with the ML estimator.

\section{CONCLUSION}

A low-complexity ML OFDM channel estimator is proposed. It relies on a deterministic model (i.e., no statistical information on the channel) that takes the finite delay spread of the channel into account, which is linked to the frequency correlation of the channel. Our ML estimator can be interpreted as a translation of some initial estimate of the frequency response of the channel to the time domain, followed by a linear transformation of this channel impulse response, and retranslation to the frequency domain. This interpretation leads to low-complexity algorithms, derived by combining the partial (I)FFTs involved and a small weighting matrix. Comb spectrum due to PSAM allows further pruning of the FFTs.

Based on theoretical grounds that shed a new light on former solutions [2], [3], the proposed algorithms have a significantly lower complexity than the low-rank approximation of the LMMSE estimator [1], while being optimal, up to possible modeling errors.

\section{ACKNOWLEDGMENT}

The authors gratefully acknowledge their colleagues who successfully implemented the ML estimator on an OFDM application-specified integrated circuit.

\section{REFERENCES}

[1] O. Edfors, M. Sandell, J. J. van de Beek, S. K. Wilson, and P. O. Börjesson, "OFDM channel estimation by singular value decomposition," IEEE Trans. Commun., vol. 46, pp. 931-939, July 1998. 
[2] G. G. Raleigh and V. K. Jones, "Multivariate modulation and coding for wireless communication," IEEE J. Select. Areas Commun., vol. 17, pp. 851-860, May 1999.

[3] P. Vandenameele, L. Van der Perre, M. Engels, B. Gyselinckx, and H. De Man, “A combined OFDM/SDMA approach," IEEE J. Select. Areas Commun., vol. 18, pp. 2312-2321, Nov. 2000.

[4] J. A. C. Bingham, "Multicarrier modulation for data transmission: An idea whose time has come," IEEE Signal Processing Mag., vol. 28, pp. 5-14, May 1990.

[5] H. V. Sorensen and C. S. Burrus, "Efficient computation of the DFT with only a subset of input or output points," IEEE Trans. Signal Processing, vol. 41, pp. 1184-1200, Mar. 1993.
[6] S. He and M. Torkelson, "Computing partial DFT for comb spectrum evaluation," IEEE Signal Processing Lett., vol. 3, pp. 173-175, June 1996.

[7] B. Debaillie, B. Come, W. Eberle, S. Donnay, M. Engels, and I. Bolsens, "Impact of front-end filters on bit-error rate performances in WLAN-OFDM transceivers," in Proc. IEEE Radio Wireless Conf., Boston, MA, Aug. 2001, pp. 193-196. 